\title{
TAILOR-MADE NETWORKS: SYNTHESIS AND PROPERTIES
}

\author{
P. Rempr, J. Herz, G. Hild and C. Picot \\ Centre de Recherches sur les Macromolécules, 67083 Strasbourg-Cedex, \\ France
}

\begin{abstract}
Methods were developed to synthesize homogeneous networks characterized by a well-known (and nearly constant) length of their linear elements, and in some cases by the constant functionality of the branch points. These methods are based on reacting the chain ends of 'precursor' polymer molecules to bind them with branch point sites. Crosslinking does not involve any major change in the segment concentration, nor does it lead to solvent expulsion. Thus the gel point has a physical significance.

These tailor-made networks were studied thoroughly using various techniques: equilibrium swelling and uniaxial compression measurements, as well as equilibrium partition coefficients, the latter being meant to characterize the average pore size of the gels by comparison with the molecular dimensions of linear polymers of the same nature.

The experimental results were compared with the predictions of the existing theories, assuming that all 'precursor chains' have become elastic chains upon crosslinking (i.e. chains attached by their two ends to two different branch points), that they behave as Gaussian chains and that the fluctuations in the number of chains connected with one branch point are small within a sample.

Results of equilibrium swelling measurements are in satisfactory agreement with theoretical predictions, and there is also a satisfactory fit for uniaxial deformation experiments. However, the relation between the concentration of segments upon crosslinking and the so-called 'memory term', which characterizes the relaxation state of the elastic chains in the network, has to be discussed thoroughly: results of the swelling and uniaxial compression measurements are used, as well as some very recent results obtained by neutron smallangle scattering on partly deuterated polystyrene networks. The results so far obtained by this technique support expectations as to what concerns the homogeneity of the gels, and they also give indications on the average distance between branch points in the gel.

Finally, the results obtained on measuring the equilibrium partition constants in a ternary system-solvent-linear polymer-gel-are also discussed, as they can be used as a measure of the pore size distribution within a gel. Account must be taken, however, of the partial deswelling of the tailor-made gels in the presence of a linear polymer (even one of the same chemical nature). Osmotic effects cannot account for the total effect observed.
\end{abstract}

The existing theories of equilibrium swelling of polymer networks and of rubber elasticity are based upon some assumptions concerning the gel itself: 
(1) It must be homogeneous, macroscopically as well as microscopically: no syneresis should occur during its formation, and the segment density as well as the branch point density should be the same throughout the network sample.

(2) It must be ideal, which means that each linear chain element must be connected with two different branch points, and that two branch points should be connected by one chain only.

(3) It should also be Gaussian; the dimensions of each chain should deviate very little from what they would be if they followed Gaussian statistics.

Furthermore it is obviously useful to consider that the fluctuations in the length of the linear chain elements within one given sample be as small as possible, and that the average length of these elements be determined accurately.

The methods of preparation of crosslinked gels which have been used in most cases until now can be classified into two groups: those involving radical (random) copolymerization of two monomers ${ }^{1}$, one of which is bifunctional and present in smaller proportion, and those involving bridge formation between preexisting linear polymer chains ${ }^{2,3,15}$. Both methods involve random processes and lead therefore to polymer networks which do not satisfy the conditions referred to above. In particular, the fluctuations over the length of the linear chain elements are quite large, and the homogeneity of the network is not satisfactory.

To get more adequate samples to investigate, we have developed ${ }^{4,5}$ for the past five years several methods of preparation of so-called tailor-made networks which should at least satisfy three conditions:

(1) Homogeneity: no syneresis, constant segment density and constant branch point density throughout the gel.

(2) Ideality, which implies absence of pendant chains and of loops, and low probability of double connections between two branch points.

(3) Adequate choice of the length of the linear chain elements - which means that the porosity of the network can be chosen arbitrarily-and low fluctuations around their average length.

Thus we can synthesize homologous series of networks meeting the same requirements and differing only by the average length of the chain elements between crosslinks. In some cases we went even one step further ${ }^{6}$ : control and constancy of the functionality of the branch points, i.e. the number of chains connected with one crosslinking site.

\section{SYNTHESIS OF MODEL NETWORKS}

The three methods which were developed to achieve this goal have already been described elsewhere in detail ${ }^{4-8}$. Let us point out briefly the main features of these methods.

(1) Anionic block copolymerization of styrene and divinylbenzene (DVB) using a bifunctional initiator

In the first step a bifunctional linear precursor polymer fitted at both ends with 'living' sites is obtained. Its molecular weight can be chosen arbitrarily 
and its polydispersity remains low. In the second step the solution of the precursor is adequately mixed with a small amount of divinylbenzene, which on polymerization yields the crosslinks, since each nodule of polydivinylbenzene will be connected simultaneously with several chain ends. Owing to adequate choice of solvent and temperature and to efficient mixing, the obtained gels are homogeneous and close to ideality. This latter characteristic can be checked for by prolonged extraction of precursor chains: if $a$ is the percentage of precursor extracted assuming for both ends equal reactivity, the probability for a chain to remain pendant within the network is given by $2 a^{\frac{1}{2}}\left(1-a^{\frac{1}{2}}\right)$. Since $a$ is always very low $(<0.01)$, the percentage of pendant chains in the gel should remain very small.

This method has been used for preparation of several different gel systems, including diolefine, vinylpyridine ${ }^{5}$ and methacrylic esters ${ }^{9}$, the difunctional monomer used to crosslink the precursor being divinylbenzene, or ethylene dimethacrylate when higher electroaffinity is required to achieve fast initiation of the difunctional monomer.

The main drawback of this method of synthesis is that it does not provide any control of the functionality, $f$, of the branch points-the number of chains attached to one given crosslinking point-nor does it prevent fluctuation in the value of $f$ occurring within a given network. It was established, however, that the higher the amount of DVB used per living end ${ }^{4}$, the tighter the structure of the network, which means that on increasing the size of the nodules of poly-DVB one also increases their average functionality.

\section{(2) Anionic polymerization followed by deactivation with a \\ plurifunctional deactivator}

This method has been tried by several authors, and requires exact stoichiometry. The main difficulty of this method is that in many cases the functions of one given plurifunctional deactivator do not exhibit equal reactivities. We were able, however, to show that in trisallyloxytriazine (TT) ${ }^{6}$ all three functions react equally well with unshielded carbanions such as styryl or isoprenyl. With shielded carbanions, such as diphenylmethyl anions ${ }^{7}$, two out of three functions still react. If carried out under appropriate conditions, the reaction of a bifunctional 'living' polystyrene with the exact amount of TT yields a network in which the branch points should be trifunctional, and in which the linear chain elements are identical in length - to a first approximation. The gels obtained by this method also exhibit satisfactory homogeneity, and should be close to ideality, in spite of the possible occurrence of some loop formation ${ }^{3,11}$, i.e. chains which are attached by their two ends to the same branch point.

Other experiments were carried out with a tetrafunctional deactivator, obtained by reaction of disodio-1,4-tetraphenyl-1,1,4,4-butane with TT, and the chosen formula is as follows:<smiles></smiles>

Here also the gels obtained are homogeneous and close to ideality. 


\section{(3) Siloxane networks}

The third method which was developed at our Institute is based upon a well-known reaction of silane functions added to allyl groups ${ }^{12}$

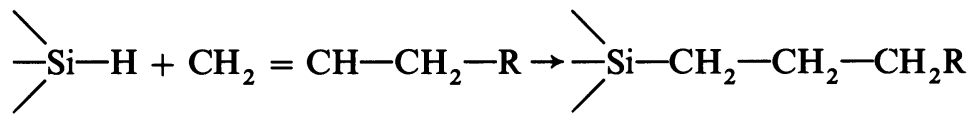

This addition proceeds with quantitative yields under the influence of chloroplatinic acid. We applied this reaction to polydimethylsiloxanes fitted at both ends of their chain with silane functions*, and we caused these polymers to react with stoichiometric amounts of tetraallyloxyethane, a tetrafunctional compound. This reaction was carried out either in bulk or in the presence of an inert diluent; gelification of the reaction medium takes place readily. The structure of the gel should be close to ideality, as in the preceding case, although loops may be formed to a small extent. The molecular weight distribution of the PDMS precursors, which determines the fluctuations in length of the linear chain elements in the network, is not as sharp as in the case of anionically obtained precursors, but the obtained gels can be considered as model networks, since both the average distance between crosslinks and the functionality of the latter are determined and remain the same throughout the gel.

Similar methods of preparation of model networks have been developed recently in various laboratories. Of particular interest is the method used by Pinazzi and his co-workers ${ }^{13}$, who synthesized model networks from short-chain precursor polymers fitted with $\mathrm{OH}$ terminal groups and plurifunctional isocyanate compounds. Stepto and his co-workers ${ }^{14}$ started from star-shaped polyethylene oxides fitted with $\mathrm{OH}$ terminal functions and caused the latter to react with a diisocyanate. If exact stoichiometry is used, the branches 'dimerize', whereas the functionality of the branch points is that of the precursor stars (here $f=3$ ).

\section{EQUILIBRIUM VOLUME-SWELLING OF A MODEL NETWORK}

If we assume our networks to be ideal, it means that all of the precursor chains - the molecular weight of which is $M$-have become elastic chains during the crosslinking process. The number of elastic chains per unit volume is therefore given by

$$
v=1 / M \bar{v}^{0}
$$

where $\bar{v}^{0}$ is the specific volume of the dry network. To calculate the volume degree of swelling, $Q$, at equilibrium-which is the reciprocal of the volume segment concentration, $v$-one sets the chemical potential of the solvent, $\mu_{1}$, to be identical inside and outside the swollen gel ${ }^{15,16}$, which gives

$$
\mu_{1}-\mu_{10}=R T \ln (1-v)+v+\chi v^{2}+R T \bar{V}_{1} v\left[A h^{\frac{2}{3}} v^{\frac{1}{3}}-B v\right]=0
$$

In this expression the first term expresses the energy of dilution and the second characterizes the elasticity of the network. Here $\chi$ is the segment-

* We should like to express our appreciation to the Rhone-Poulenc Co. for sending us wellcharacterized samples of PDMS with silane end groups. 
segment interaction parameter is the chosen swelling solvent, $T$ is the absolute temperature, $R$ is the gas constant, $\bar{V}_{1}$ is the molar volume of the solvent, $A$ and $B$ are constants and $h$ is the so-called 'memory term', which will be discussed in more detail later. $A$ is usually taken as one ${ }^{16,17}$, whereas $B$ is related to the average functionality of the branch points by the following relation $^{15,16}$ :

$$
B=2 / f
$$

The above equation can thus be written ${ }^{10}$ as

$$
v=\frac{1}{M \bar{v}^{0}}=\frac{\ln (1-v)+v+\chi v^{2}}{\overline{V_{1}}\left[(2 / f) v-h^{\left.\frac{2}{3} v^{\frac{1}{3}}\right]}\right.}
$$

It is possible now to study the swelling behaviour of homologous gels, i.e. of gels prepared under the same conditions except for one experimental parameter, such as the molecular weight of the precursor or the functionality of the branch points.

The latter point is not very easy to deal with, since network synthesis via block copolymerization of styrene and DVB does not yield any precise knowledge of the functionality of branch points. In Table 1 the swelling degrees in benzene of polystyrene gels made by two of the above-described techniques are compared. It can be seen that on increasing the amount of DVB per living end the network becomes tighter. But when the proportion of DVB used is not more than three molecules per living end, the degree of swelling of the gels is not very different from that observed for gels obtained by the other method, in which the functionality of the branch points is equal to three.

Another parameter has to be considered here: the over-all concentration at the gel point. One might think that in cases in which the functionality of the branch points is determined by that of a reagent (methods 2 and 3) and the molecular weight of the precursor is known, the over-all concentration

\begin{tabular}{|c|c|c|c|}
\hline \multicolumn{2}{|c|}{ Type A (3 DVB/LE) } & \multicolumn{2}{|c|}{ Type $\mathbf{B}(f=3)$} \\
\hline $\begin{array}{c}M \\
7600\end{array}$ & $\begin{array}{l}Q_{\mathrm{C}_{6} \mathrm{H}_{6}} \\
10.2\end{array}$ & $\begin{array}{c}M \\
7000\end{array}$ & $\begin{array}{l}Q_{\mathrm{C}_{6} \mathrm{H}_{6}} \\
11.0\end{array}$ \\
\hline 10600 & 12.6 & 10600 & 14.25 \\
\hline 14500 & 12.9 & 13000 & 15.1 \\
\hline 19700 & 15.8 & 19900 & 20.25 \\
\hline 31500 & 24.3 & 30300 & 25.6 \\
\hline \multicolumn{4}{|c|}{ Type A } \\
\hline$M$ & $\mathrm{DVB} / \mathrm{LE}$ & $Q_{\mathrm{C}_{6} \mathrm{H}_{6}}$ & $Q_{\mathrm{C}_{6} \mathrm{H}_{12}}$ \\
\hline \multirow{3}{*}{25000} & 3 & 17.4 & 3 \\
\hline & 6 & 14.9 & 2.85 \\
\hline & 10 & 12.5 & 2.6 \\
\hline
\end{tabular}

Table 1. Equilibrium degree of swelling of polystyrene model networks 


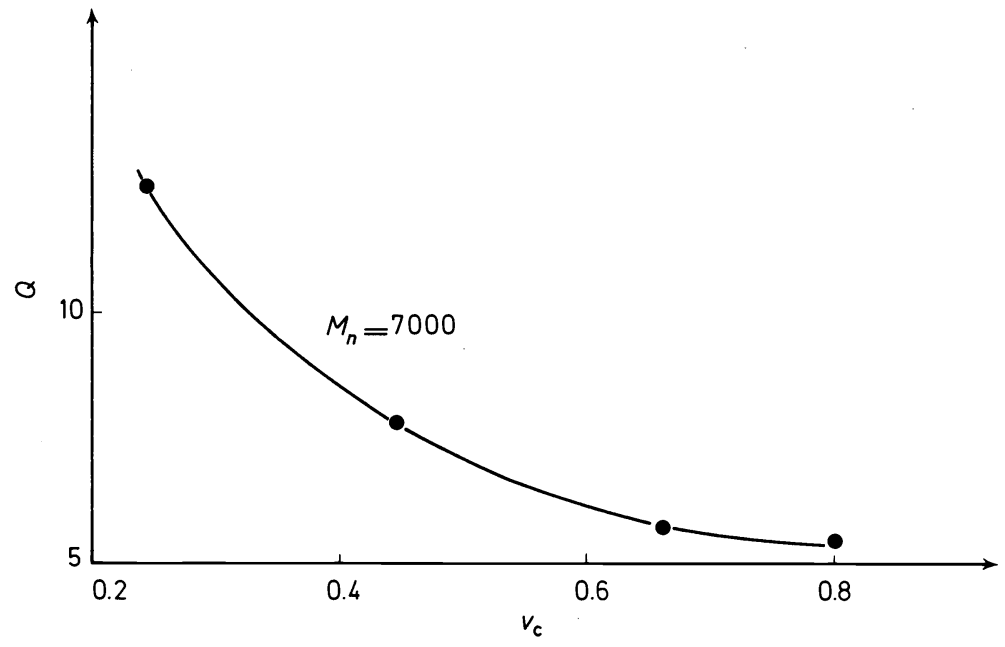

Figure 1. Variation of equilibrium degree of swelling, $Q$, for PDMS networks as a function of the concentration at which they have been obtained

of the medium should not affect the swelling behaviour of the gel, provided no syneresis has occurred. In fact things are not as simple as that. Figure 1 shows a plot of the equilibrium swelling degree of PDMS gels against the concentration, $v_{c}$, at which they have been prepared. Obviously the variation of $Q$ against $v_{c}$ for each given precursor is far above experimental error. To account for this, it can be assumed that at higher concentrations the probability of cyclization is greatly reduced and the gels come closer to ideality. On the other hand, the probability of entanglement formation is also increased, although this argument would be stronger for higher molecular weights of the precursors.

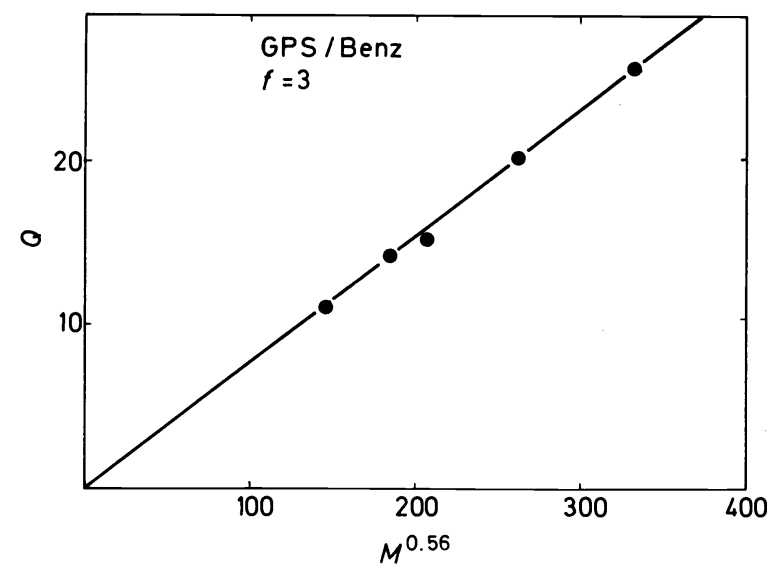

Figure 2. Variation of the equilibrium degree of swelling, $Q$, of polystyrene networks $(f=3)$ versus $M^{0.56}$, where $M$ is the average molecular weight of the precursor. All gels have been obtained under identical conditions 
Many attempts have already been made to relate the degree of swelling with the molecular weight of the precursor chains, for a series of homologous networks.

In a first very rough approach ${ }^{10,18}$ we showed that provided

$$
Q \gg 1 \text { and } Q>(2 / f)^{\frac{3}{2}} 1 / h
$$

the degree of swelling should be a linear function of $M^{\frac{3}{3}}$. Curiously enough, the same type of dependence is found for polystyrene networks with $f=3$ (Figure 2): in this case the above inequality is obviously not satisfied.

To obtain a more precise relationship between $M$ and $Q$ in the case of networks obtained by anionic block copolymerization, it was attempted to take into consideration the possible variation of the interaction parameter, $\chi$, with the segment concentration in the gels, the parameters involved being the 'memory term', $h$, the concentration upon crosslinking, $v_{\mathrm{c}}$, and the functionality, $f$. It can be seen from the plot of $M$ against $Q$ (Figure 3 ) that

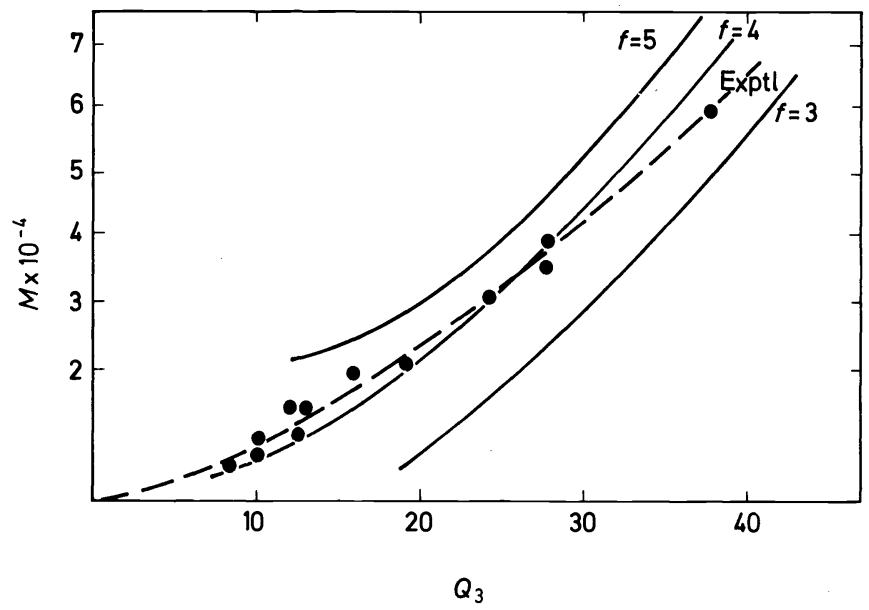

Figure 3. Relation between $M$ and $Q$ for a homologous series of polystyrene networks. Theoretical curves are obtained under the assumption of $\chi=\chi(c) ; h=0.354 v_{\mathrm{c}}$; The dashed line is the experimental curve

the experimental curve does not fit very satisfactorily with the calculated curves, each of them corresponding to a given value of $f^{19,20}$. It follows that an average functionality of four or five is plausible; however, this type of relationship is of no great use so long as the problem of the relation between $h$ and $v_{\mathrm{c}}$ is not settled.

In the case of PDMS networks, obtained at high concentration, and usually of low swelling degrees (especially in toluene), it was found that $Q$ is proportional to $M^{\frac{3}{3}}$ (Figure 4). In toluene PDMS chains behave as in a theta solvent, which means that $\chi$ is close to one-half. In the Dušek-Prins equation $^{16}$ the logarithm can be replaced by the three first terms of its 


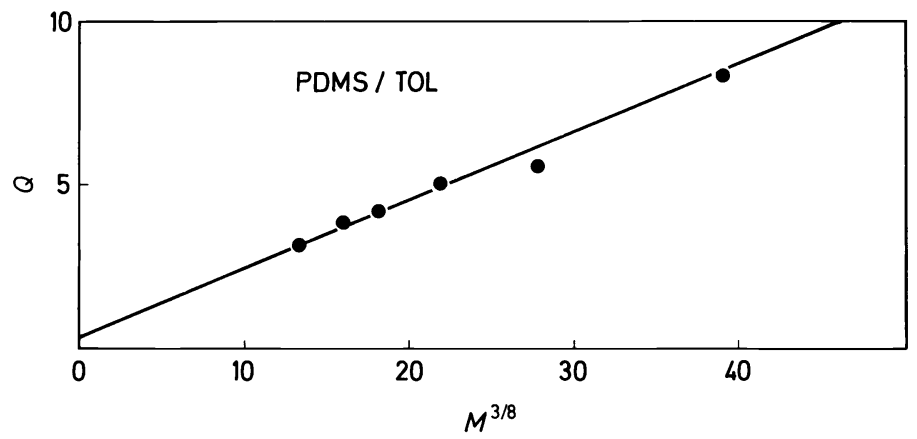

Figure 4. Relation between $Q$ and $M$ for a homologous series of PDMS networks prepared in toluene at a concentration of $67 \%$

development, which leads to

$$
Q=\left(\frac{2}{3 \bar{V}_{1}}\right)^{\frac{3}{8}} \times \frac{\bar{v}^{0 \frac{3}{8}}}{v_{\mathrm{c}}^{\frac{2}{2}}\left\{1-\left(1 / Q v_{\mathrm{c}}\right)^{\frac{2}{3}}\right\}^{\frac{3}{8}}} \times M^{\frac{3}{8}}
$$

Obviously the term in braces in the denominator still contains $Q$, but its variation is of very small amplitude, so long as $Q$ stays between 3 and 8 , as it does for our PDMS networks swollen in toluene.

\section{UNIDIRECTIONAL STRAIN}

If an equilibrium swollen gel is submitted to a unidirectional deformation which will change its length from $L_{\mathrm{i}}$ to $L$, whereby its degree of swelling changes from $Q_{\mathrm{i}}$ to $Q$, the applied stress $\sigma$ (force per unit dry unstrained cross-section) is related with the extension ratio, $\Lambda=L / L_{\mathrm{i}}$, by the following equation $^{16,21}$ :

$$
\sigma=A v R T Q_{\mathrm{i}}^{+} h^{\frac{3}{3}}\left[\Lambda-\left(Q / Q_{\mathrm{i}}\right) \Lambda^{-2}\right]
$$

Obviously, if constant volume is assumed, $Q$ and $Q_{\mathrm{i}}$ are identical and the above expression can be reduced to

$$
\sigma=A v R T Q^{\frac{1}{3}} h^{\frac{2}{3}}\left[\Lambda-\Lambda^{-2}\right]
$$

However, except for measurements carried out on unswollen (dry) networks - above the glass transition temperature - the volume of the gel under stress cannot be assumed to remain constant.

Some authors ${ }^{22,23}$ have tried to avoid taking this deswelling into consideration by carrying out the uniaxial compression measurements very quickly, but it is not established whether such experiments really lead to equilibrium measurements.

In fact, even low stresses have as a result a noticeable change in volume ${ }^{24}$, and in all our experiments we have taken into account this deswelling effect, using the following relation ${ }^{25}$ to characterize the amount of deswelling, $Q / Q_{\mathrm{i}}$, observed for a deformation $\Lambda$ : 


$$
Q / Q_{\mathrm{i}}=\Lambda^{\frac{1}{2}}\left[1+\frac{1}{2} B\left(\Lambda^{\frac{1}{2}}-1\right) Q_{\mathrm{i}}^{-\frac{2}{3}} h^{-\frac{2}{3}}\right]
$$

This expression is an approximation which fits satisfactorily in many cases. Its validity has been tested for our own gels.

The modulus $G^{*}$ is defined as:

$$
G^{*}=\sigma /\left[\Lambda-\left(Q / Q_{\mathrm{i}}\right) \Lambda^{-2}\right] Q_{\mathrm{i}}^{2}
$$

Thus it is of interest to study the variation of $G^{*}$ with the molecular weight of the linear elements of the network:

$$
G^{*}=A R T v h^{\frac{2}{3}}
$$

If the network is ideal, one has obviously

$$
v=1 / M \bar{v}^{0}
$$

and if the 'memory term' does not change with molecular weight, one could expect a linear variation of $G^{*}$ against $\boldsymbol{M}^{-1}$.

The experimental results ${ }^{26}$ obtained on homologous series of PDMS networks are shown in Figures 5 and 6. The samples prepared in toluene (a

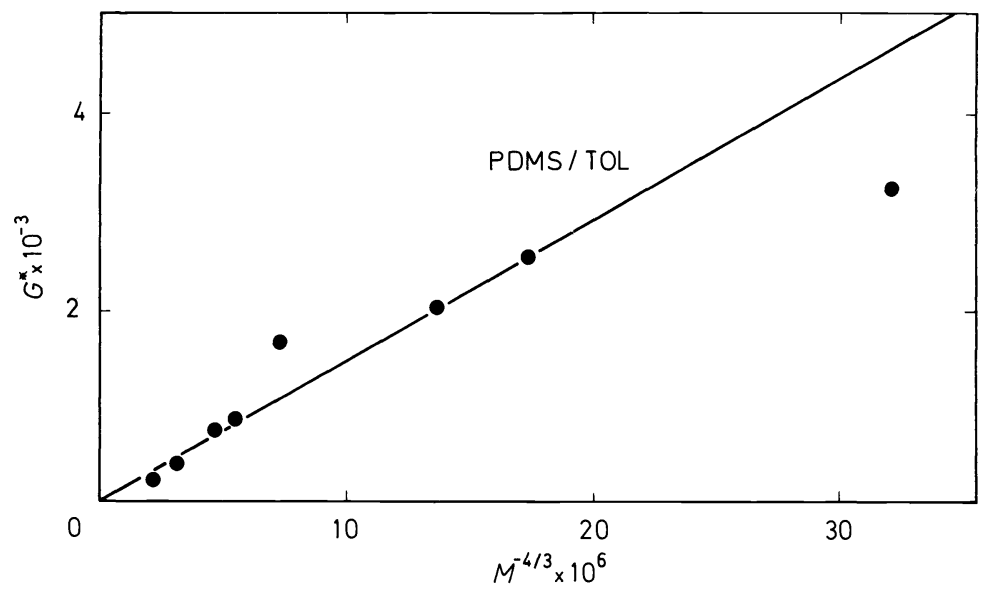

Figure 5. Variation of the unidirectional stress moduli, $G^{*}$, for PDMS networks swollen in toluene versus $M^{-\frac{4}{3}}$. Synthesis of the networks was carried out in toluene also

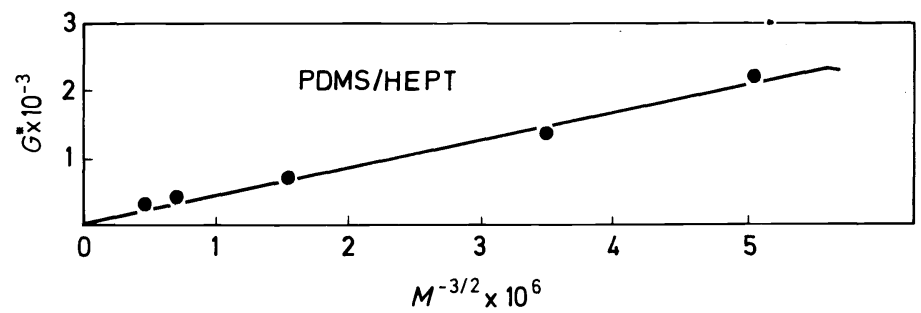

Figure 6. Variation of the unidirectional stress moduli, $G^{*}$, for PDMS networks swollen in heptane versus $M^{-\frac{3}{2}}$. Synthesis of the networks was carried out in heptane also 
theta solvent for PDMS) have been swollen in the same solvent, and it is shown that, provided the networks were obtained at sufficiently high concentrations, $\boldsymbol{G}^{*}$ is a linear function of $\boldsymbol{M}^{-\frac{4}{3}}$. When heptane (a thermodynamically good solvent for PDMS) is used as diluent for the synthesis of the networks, and then as a swelling agent, $G^{*}$ is found to be proportional to $M^{-\frac{3}{2}}$; when the networks have been obtained at higher dilution, the slope of the lines is less and even this proportionality law is no longer obeyed.

To account for these relationships one has to take into consideration the variation of the 'memory term', $h$, with molecular weight, which will be dealt with later.

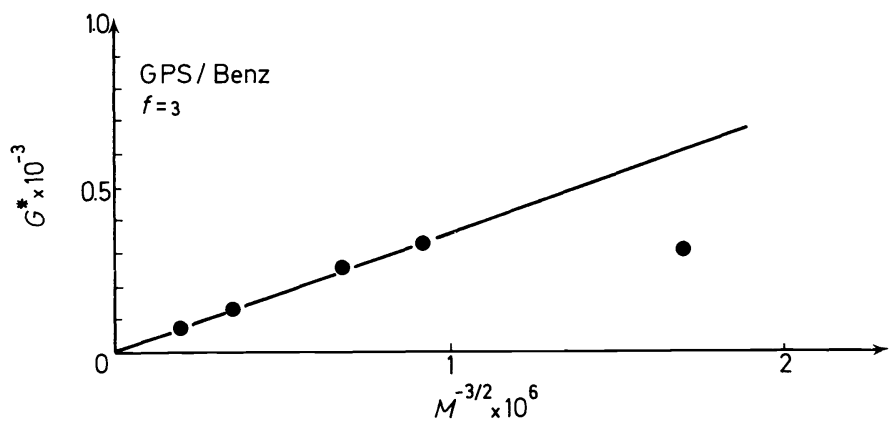

Figure 7. Variation of $G^{*}$ versus $M^{-\frac{3}{2}}$ for a homologous series of polystyrene networks $(f=3)$

It is of interest to note that polystyrene networks synthesized according to method (2), and thus exhibiting trifunctional branch points, have been also investigated in benzene: the obtained values of $G^{*}$ are found to be proportional to $M^{-\frac{3}{2}}$ (Figure 7).

\section{PARTITION COEFFICIENTS}

In investigating the pore size distribution within our networks, it was of interest to determine the static (equilibrium) partition coefficients of linear polystyrenes within a given network, over a wide range of molecular weights of the polystyrene samples ${ }^{27} . K_{\mathrm{s}}$ is defined as the ratio of the concentration of the linear polymer within the pores of the network to its concentration in the surrounding solution. The linear polymer and the network chains were of the same chemical nature, to avoid heterocontact interactions. The problem is to determine in which range of molecular weights the size of the linear chain molecules meets the dimensions of the pores. If the polymer molecules are very small, they may enter the pores of the swollen gel as do the solvent molecules: $K_{\mathrm{s}}$ is close to unity. If the polymer molecules are very large, they cannot enter the pores and $K_{\mathrm{s}}=0$. In an intermediate range of molecular weights the value of $K_{\mathrm{s}}$ characterizes the fraction of pores which are accessible to polymer molecules. Obviously it is necessary to use sharp 
fractions of polymer, in order to avoid complications arising from polydispersity (Figure 8).

There is, however, another difficulty arising in static partition coefficient measurements: the equilibrium degree of swelling is far below that observed in the pure solvent. To account for this fact one can first invoke osmotic effects: the polymer concentration being smaller inside the gel than outside it, some deswelling has to be expected ${ }^{28}$. Moreover this deswelling should

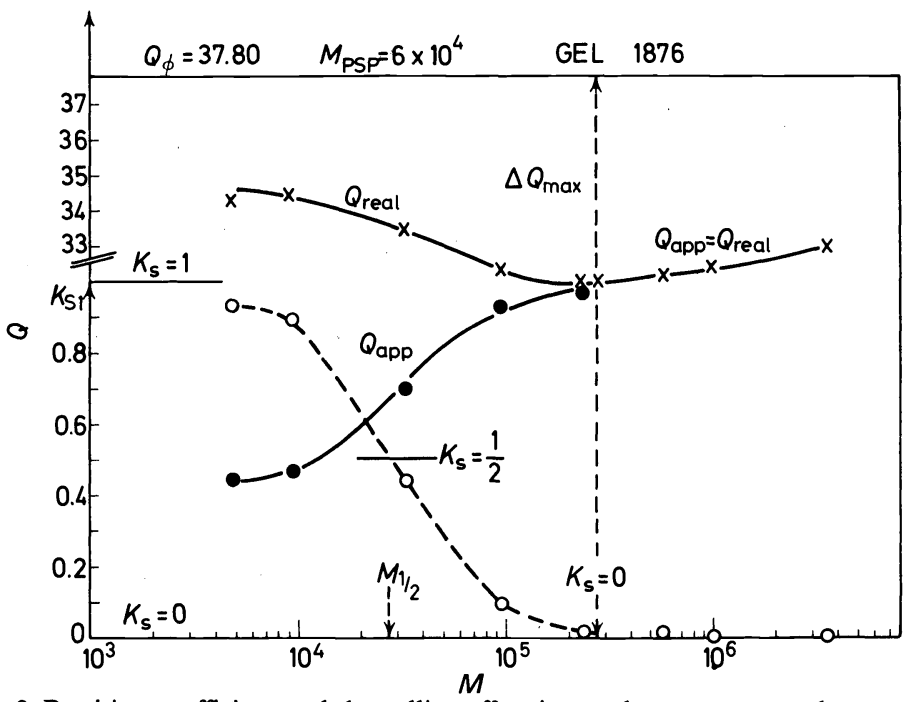

Figure 8. Partition coefficient and deswelling effect in a polystyrene network versus molecular weight of the linear polystyrene molecules

decrease with increasing molecular weight of the linear polymer. This is what is observed, but this explanation is insufficient, since it would not account foresuch large deswelling as is experimentally observed. Another explanation must be sought. According to solution measurements carried out by several authors $^{29}$, the thermodynamic quality of a solvent is decreased when it contains some polymer, even if the two polymers are of the same chemical nature. If they are not, the effect is much greater ${ }^{30}$. Thus it can be expected that benzene alone is a better swelling agent for the network than a benzene solution of a linear polystyrene, although the segment-segment interactions can be considered to be the same, regardless of whether the segments are part of the network or part of a linear chain ${ }^{27}$.

This theory is as yet quite qualitative, and further work is necessary to establish whether precise predictions can be made concerning the extent of deswelling of a network in a ternary system, solvent-linear polymer-network, with respect to the degree of swelling of the gel in the pure solvent. 


\section{DISCUSSION}

\section{Structure of the model networks}

From the experimental results described above considerable information on the structure and behaviour of the model networks has been obtained. There are, however, several points which need further discussions: (1) the 'memory term' and how to relate the structure of a network with its preparation conditions; (2) the space distribution of the branch points in the swollen network; and (3) how to determine the average functionality of the branch points.

\section{Memory-term, $h$}

By definition the 'memory term', $h$, characterizes the segment concentration in the gel under such conditions as to have all elastic chains in the relaxation state $^{16}$. Obviously the relaxation state is neither the dry stateunless the crosslinking process has taken place in the bulk-nor the state of equilibrium swelling. It has to be related with the degree of swelling which the gel exhibited upon its formation. This is why $h$ is usually referred to as the 'memory term'.

When a network is synthesized by radical copolymerization, the gel point has no physical significance, because gelification occurs at an early stage of the reaction. As the polymerization proceeds, the segment concentration of the network increases, although the volume of the gel remains unchanged, provided no syneresis occurs. In the case of our model networks, on the contrary, the crosslinking process does not involve any significant change in the segment density, because it merely consists in binding the chain ends of a precursor to crosslinking sites of functionality, $f$. The gel point has here a physical significance, and thus the 'memory term', $h$, could possibly be related with the segment concentration, $v_{c}$, at the time at which crosslinking occurred. According to Dušek and Prins ${ }^{16}$, the 'memory term', $h$, is defined by the following relationships:

$$
h^{\frac{2}{3}}=\frac{\overline{r^{2}}}{\overline{r_{0 \mathrm{~s}}^{2}}}=\frac{\overline{r^{2}} \mathrm{c}}{\overline{r_{0 \mathrm{~s}}^{2}}} \times \frac{\overline{r^{2}}{ }_{\mathrm{d}}}{\overline{r^{2}}}=\frac{\overline{r_{\mathrm{c}}^{2}} \mathrm{c}}{\bar{r}_{0 \mathrm{~s}}^{2}} \times v_{\mathrm{c}}^{\frac{3}{3}}
$$

Here $\overline{r_{0 \mathrm{~s}}^{2}}$ is the mean square dimension of a free precursor chain in the chosen solvent; $r_{\mathrm{d}}{ }_{\mathrm{d}}$ is the mean square dimension of an elastic chain in the dry network; and $\bar{r}_{\mathrm{c}}$ is the mean square dimension of the elastic chains just after the crosslinks have been introduced. Thus $v^{\frac{2}{3}}$ is equal to the ratio of $\bar{r}_{\mathrm{c}}^{2}$ to $r^{2}$, and it is experimentally accessible in the case of our model networks.

The knowledge of $h$ depends now on the evaluation of the $r^{2}{ }_{\mathrm{c}} / r_{0 \mathrm{~s}}^{2}$ ratio. Several authors ${ }^{17,31,32}$ using different approaches have assumed that upon crosslinking the mean square dimensions of the linear chain elements undergo a uniform shrinkage by a factor of approximately 2 . Accordingly, $h$ should be equal to $0.354 v_{\mathrm{c}}$. We have also used this approach in some past research work $^{19}$, and at first sight it seemed to lead to consistent results.

However, if one tries to obtain a more precise insight, it seems obvious that the $\overline{r_{\mathrm{c}}^{2}} \overline{r_{0 \mathrm{~s}}^{2}}$ ratio should not be constant: its value should depend upon the functionality, $f$, of the branch points, upon the molecular weight, $M$, of the elastic chains, and, possibly, upon the thermodynamic quality of the solvent in which crosslinking occurred, $\chi$. 
These points will be examined in more detail in the next section.

\section{Relation between functionality, $f$, and intercrosslink distance, $d$}

Let us assume that the gels we are dealing with are ideal and homogeneous. At the gel point the segment concentration (in volume) is $v_{\mathrm{c}}$. The number, $n$, of elastic chains per $\mathrm{ml}$ of the gel is equal to the number of precursor chains in the unit volume of the reaction medium, namely:

$$
n=\left(v_{\mathrm{c}} \rho / M\right) N_{\mathrm{A}}
$$

where $\rho$ is the density of the solvated chains and $N_{\mathrm{A}}$ is Avogadro's number. The number of $f$ functional branch points per unit volume is given by

$$
n_{\mathrm{b}}=(2 / f)\left(v_{\mathrm{c}} \rho / M\right) N_{\mathrm{A}}
$$

and the average volume, $V$, available per branch point is the reciprocal of $n_{\mathrm{b}}$ :

$$
V=\left(f M / 2 v_{\mathrm{c}} \rho N_{\mathrm{A}}\right)
$$

We have now to make new assumptions concerning the average (or equilibrium) positions of the branch points with respect to one another.

The use of pseudocrystalline models may be astonishing at first sight, since there is a priori no reason why the branch points should be placed in a regular fashion in the swollen (and even in the dry) network. It may also happen that the average functionality of the branch points is 5 or 7 , or another value which does not allow any regular placement in space.

However, if one considers that each branch point is attached to $f$ chains connecting it elastically with as many first neighbours distributed in space around it, one can expect some kind of order to exist, as in an ensemble of spring-suspended beads. It should be recognized that there is no absolute requirement for a branch point to be connected to all of its first neighbours, or only to its first neighbours.

Let us use, as a first example, a simple cubic lattice to schematize a network. The number of next neighbours is 6 , but this lattice can be used also for values of $f \neq 6$, taking into account that some chains may be connected with second neighbours (if $f>6$ ) or that some connections between first neighbours are missing (if $f<6$ ). If we take this model, knowing that the unit cell contains one single branch point and that the first neighbours are consequently distant by the length, $a$, of the unit cell, we can write

$$
d=a=V^{\frac{1}{3}}
$$

The same calculations have been carried out for other types of compact distribution models, obeying the condition of isotropy, each corresponding to a given average functionality of the crosslinking sites $(f=4,8,12)$ : (1) the face centered cubic lattice, the most compact unit cell $(f=12)$; (2) the body centered cubic cell $(f=8)$; and (3) the diamond-type cubic repeat unit $(f=4)$. The data concerning these models are gathered in Table 2.

Evaluation of the $\overline{r_{\mathrm{c}}^{2}} / \overline{r_{0 \mathrm{~s}}^{2}}$ ratio

In the preceding paragraph it was shown under which assumptions the 
intercrosslink distance, $d$, could be evaluated, at the gel point, i.e. using $v_{\mathrm{c}}$ as the segment concentration (by volume). Evidently $d$ is nothing else but $\left(\bar{r}_{c}^{2}\right)^{\frac{1}{2}}$, since it characterizes the root mean square end-to-end distance of

Table 2. Intercrosslink distances in various regular lattices

\begin{tabular}{lcccc}
\hline & $\begin{array}{c}\text { Simple } \\
\text { cubic }\end{array}$ & $\begin{array}{c}\text { Body centred } \\
\text { cubic }\end{array}$ & $\begin{array}{c}\text { Face centred } \\
\text { cubic }\end{array}$ & $\begin{array}{c}\text { Diamond-type } \\
\text { cubic }\end{array}$ \\
\hline Number of units per cell & 1 & 2 & 4 & 8 \\
Number of first neighbours & 6 & 8 & 12 & 4 \\
$\begin{array}{c}\text { Distance, } d \text {, between first } \\
\text { neighbours* }\end{array}$ & $a$ & $\frac{a \sqrt{3}}{2}$ & $\frac{a \sqrt{2}}{2}$ & $\frac{a \sqrt{3}}{4}$ \\
& $V^{\ddagger}$ & $3^{\frac{1}{2}} 2^{-\frac{7}{3}} V^{\ddagger}$ & $2^{\frac{1}{6}} V^{\ddagger}$ & $\frac{\sqrt{3}}{2} V^{\ddagger}$ \\
\hline
\end{tabular}

* Expressed as a function of the length, $a$, of the repeat cell, and as a function of the volume available per unit (branch point).

an elastic chain. This value should be compared with the mean square endto-end distance of the free chain, in that particular solvent.

Knowing the viscosity law of the system

$$
[\eta]=K M^{a}
$$

the mean square end-to-end distance can be calculated according to

$$
\overline{r_{0 s}^{2}}=\left(K M^{1+a} / \phi\right)^{\frac{2}{3}}
$$

where $\phi$ is Flory's universal constant $=2.7 \times 10^{21}$.

We now see that assuming a uniform shrinkage of the linear chains upon crosslinking, by a factor two, is an oversimplification, since the number of branch points formed in the medium - which depends upon their average functionality - determines the average intercrosslink distance, whereas $\vec{r}_{0 \mathrm{~s}}^{2}$ solely depends upon the molecular weight of the precursor chains. Thus we assume that concentration dependence of $\overline{r_{\mathrm{os}}}$ is negligible.

If we take as an example a polystyrene gel made anionically, in benzene at a concentration (by volume) of 0.1 , the average molecular weight of its linear chain elements being 25000 , we find for the root mean square end-to-end distance $115 \AA$. If the supposedly uniformly distributed branch points are tetrafunctional, their average distance should be $81 \AA$. If they are hexafunctional, $d=107 \AA$; if they are octafunctional, $d=129 \AA$; and if they are dodecafunctional, they are distant by $152 \AA$, in the average. This shows that the preparation conditions - and especially the amount of DVB used per living end - should have a marked influence on the 'memory term', i.e. on the amount of contraction or expansion introduced on the individual chains by the crosslinking process.

\section{EXPERIMENTAL EVIDENCE FOR REGULAR PLACEMENT OF BRANCH POINTS IN SWOLLEN NETWORKS}

To gain further information about the distribution of branch points in a swollen network, we have used neutron coherent scattering experiments. 
It is well known that both coherent and incoherent scattering cross-sections are widely different between hydrogen and deuterium and that it is possible to measure the radius of gyration of a deuterated polymer molecule in a non-deuterated polymer matrix, or vice versa ${ }^{33,34}$. Here our goal was to try to establish first whether the space distribution of the crosslinks in a given network is of sufficient regularity to lead to diffraction peaks, which can be handled according to Bragg's law. For the purpose of these neutron scattering experiments we have synthesized networks with 'labelled' branch points, and measured the angular scattering distribution of neutrons for these gels in bulk and in the equilibrium state. This will be briefly described in the last section of this article.

\section{Synthesis of model networks with labelled branch points}

The easiest method would have been to block copolymerize styrene with perdeuterated divinylbenzene, according to method (1), as described above. But perdeuterated DVB was not readily available, and we proceeded in a different way.

In a first step styrene is polymerized anionically-with a bifunctional initiator-to a bifunctional living precursor of known molecular weight and of sharp molecular weight distribution.

Next perdeuterated styrene is added to the solution of the precursor in such an amount that about six deuterated monomer units are located at each chain end. The precursor molecules remain 'living' and little polydisperse.

In a third step some DVB is added to the reaction medium, and after a little while crosslinking takes place. One can thus expect that each branch point, connecting $f$ precursor chain ends (which have become elastic chains upon crosslinking), is surrounded by $6 f$ deuterated styrene units, whose coherent scattering cross-section is quite different from that of the nondeuterated units of the elastic chains.

\section{Results of the neutron scattering experiments}

The major part of the neutron scattering experiments was carried out at the Langevin-Von Laue Institute in Grenoble, and used neutrons produced in the high-flux reactor; the wavelength is of the order of $7 \AA$. The apparatus (D11) was constructed by Ibel ${ }^{35}$. Experimental procedure and treatment of the results will be described elsewhere ${ }^{36,37}$.

The 'labelled' gels gave very characteristic scattering patterns exhibiting a rather sharp peak (Figure 9). Applying Bragg's law enables us to calculate the characteristic distance in the gels, which is called $d$ in Table 3.

In Table 3 the experimental $Q$ - and $d$-values are given for three gels obtained under exactly identical experimental conditions: over-all volume concentration, ten per cent; $10 \mathrm{~mol}$ DVB per living end; temperature, $35^{\circ} \mathrm{C}$. These gels differ only by the molecular weight of the precursor polystyrene. A fourth gel of the same series $(M=8000)$ has been synthesized and studied by neutron scattering. No peak could be found in the diffraction patterns. The lack of any regular spatial distribution of the branch points may be due either to the size of the DVB nodules, as compared with that of the linear elastic chains, or, more likely, to the occurrence of syneresis during the preparation of the network. It was found that its maximum degree of swelling in benzene is 
about 8 , whereas the volume concentration at the gel point $v_{\mathrm{c}}$ corresponds to a degree of swelling $Q_{\mathrm{c}}=10$.

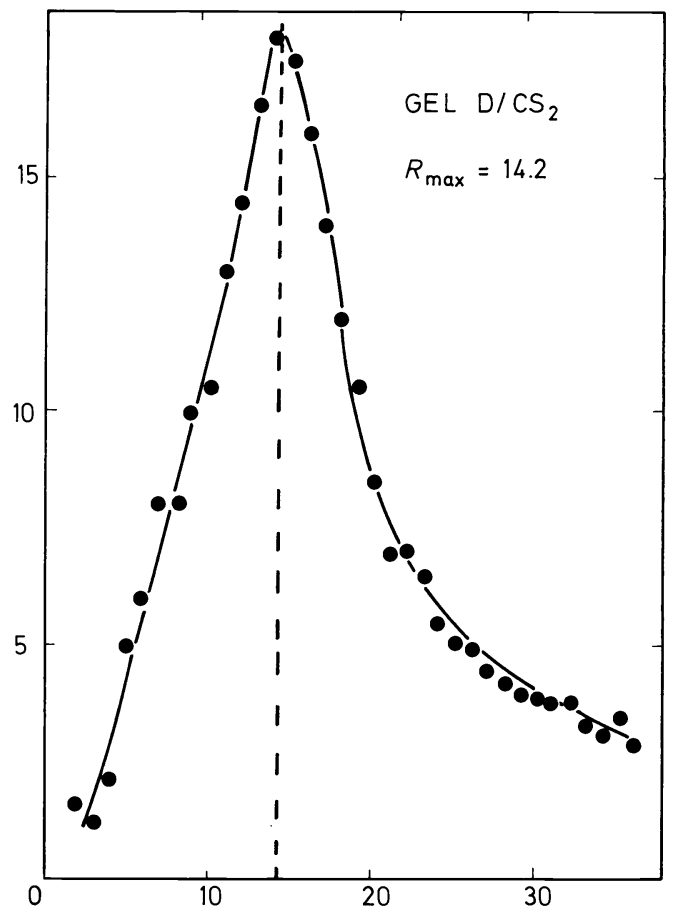

Figure 9. Example of neutron small-angle diffraction peak

Table 3. Neutron scattering data on 'labelled' model networks

\begin{tabular}{|c|c|c|c|c|c|c|c|c|c|}
\hline \multirow[t]{2}{*}{ Solvent } & \multicolumn{3}{|c|}{ Gel F, $M=21000$} & \multicolumn{3}{|c|}{ Gel D, $M=27000$} & \multicolumn{3}{|c|}{ Gel G, $M=42000$} \\
\hline & $Q$ & $d$ & $d \times Q^{-\frac{1}{3}}$ & $Q$ & $d$ & $d \times Q^{-\frac{1}{5}}$ & $Q$ & $d$ & $d \times Q^{-\frac{1}{5}}$ \\
\hline Dry & 1 & $(80) \dagger$ & 80 & 1 & 90 & 90 & 1 & 109 & 109 \\
\hline $\mathrm{C}_{6} \mathrm{H}_{12}$ & 3.2 & 126 & 85 & 2.7 & 137 & 98 & 3.2 & 158 & 107 \\
\hline $\mathrm{CS}_{2}$ & 11.4 & 165 & 73 & 11.5 & 193 & 86 & 7.4 & 227 & 88 \\
\hline $\mathrm{C}_{6} \mathrm{H}_{6}$ & & & & 12.6 & 211 & 91 & & & \\
\hline BuPh* & 4.4 & 138 & 84 & 6.6 & 144 & 77 & 7.6 & 198 & 101 \\
\hline
\end{tabular}

* Butylphtalate.

† Extrapolated value.

Obviously it is necessary to check first whether these measurements are significant: to do this we have calculated $d \times Q^{-\frac{1}{3}}$, which should remain constant for any given gel. Since swelling is isotropic, all distances in a swollen gel should be $Q^{\frac{5}{3}}$ times greater in the swollen state than in the dry network. The result of this calculation is satisfactory if account is taken of the rather low accuracy of some of the experiments. 
Next it can be noted that as the molecular weight of the elastic chains rises, the distance between crosslinks increases, the increase being much greater when the gel is swollen in a good solvent than when in the dry state or swollen in cyclohexane (a theta solvent).

It is also of interest to evaluate the functionality of the crosslinks, using the method described above. The results of this calculation are shown in Table 4 .

Table 4. Calculation of the functionality of the crosslinking sites

\begin{tabular}{lcccccccc}
\hline \multirow{2}{*}{ Gel } & $M$ & \multicolumn{9}{c}{$d, \AA$} & & \\
\cline { 3 - 6 } & & $f=4$ & $f=6$ & $f=12$ & $f=18$ & $\exp$ & & \\
\hline F & 21000 & 35 & 46.5 & 65 & 75 & 80 & 100 & 80 \\
D & 27000 & 38 & 50 & 71 & 81 & 90 & 114 & 87 \\
G & 42000 & 44 & 58.5 & 83 & 94 & 109 & 142 & 109 \\
\hline
\end{tabular}

The values of $d$ one would expect for $f=4$ or 6 or 12 are calculated by using the corresponding cubic models. The face centered cubic lattice was also used for calculation with $f=18$. In the last column the root mean square end-to-end distance of the free chains in cyclohexane is indicated; it was shown recently ${ }^{36}$ that the dimensions of a chain molecule in bulk is very close to those observed under theta conditions.

From Table 4 it follows that the functionality of the branch points must be quite high. This is due to the relatively high proportion of DVB which was added to the precursor solution $(10 \mathrm{DVB} / \mathrm{LE})$ upon synthesis of these gels.

In the dry state the dimensions of the elastic chains are of the same order of magnitude as those of the corresponding free chains, as can be seen from the three last columns of Table 4. The root mean square end-to-end distance $\left(r_{0}^{2}\right)^{\frac{1}{2}}$ of the precursor is somewhat higher than the corresponding values of $d$ in the networks, but if instead of $\left(r_{0}^{2}\right)^{\frac{1}{2}}$ one uses the mean value of the endto-end distance $\bar{r}$ the fit is really excellent.

When the gel is swollen in a good solvent, as in benzene or carbon disulphide, the isotropic expansion of the network is so large that the elastic chains are in any case much more extended in the network than the precursor chains in solution in the same solvent. As an illustration let us consider the gel D: the root mean square end-to-end distance of the precursor $(M=$ 27000 ) in benzene is $124 \AA$. The intercrosslink distance, $d$, found in benzene is $211 \AA$.

In a theta solvent, such as cyclohexane, where the degree of swelling is rather low, there is also a slight expansion of the elastic chains with respect to their dimensions in the same solvent, as free chains.

Let us now go back to the crosslinking process itself. If we assume isotropic expansion, we can calculate the intercrosslink distance, $d$, for any degree of swelling. We can thus choose the swelling degree, $Q_{\mathrm{c}}$, that corresponds to the segment concentration, $v_{\mathrm{c}}$, at which crosslinking occurred. For the gel $\mathrm{D}, Q_{\mathrm{c}}=10$, which leads to a value of approximately $190 \AA$ for $d$. The root mean square end-to-end distance of the precursor chains in benzene is 
$124 \AA$. It can be concluded that the chains expand upon crosslinking. The magnitude of this expansion depends of course upon the functionality of the branch points, which is obviously quite high in the above-quoted case.

The case of PDMS, which was discussed in an earlier section, is quite different for two reasons: the crosslinking reaction is carried out at very high concentrations and the functionality of the branch points is known and quite low $(f=4)$. From Table 5 one can see that under the assumption of regular placement of the branch points, and taking into account the viscosity laws of PDMS in toluene and in heptane, respectively, there is an appreciable shrinkage of the chain dimensions upon crosslinking.

Table 5. Intercrosslink distance and root mean square end-to-end distances in PDMS

\begin{tabular}{cccc}
\hline$M$ & $d$ & $\left.\overline{\left(r_{0}^{2}\right.}\right)^{\frac{1}{2}}$, toluene & $\left(\overline{r_{0}^{2}}\right)^{\frac{1}{2}}$, heptane \\
\hline 4500 & 24 & 40 & 48 \\
8700 & 30 & 56 & 70 \\
17500 & 39 & 79 & 105 \\
\hline
\end{tabular}

However, in the case of PDMS, owing to the fact that $f$ is known and constant throughout the gel-if loops are neglected-it is possible to check for the experimental relationships between $G^{*}$ and $M$. It is established that $G^{*}$ is proportional to $v h^{\frac{3}{3}} \cdot v$ is given by $\left.v=1 / M \bar{v}^{0}\right)$ and $h^{\frac{2}{3}}$ is proportional to $d^{2} / \overline{r_{0}^{2}}$. We have shown earlier that $d$ is a function of $M^{\frac{1}{3}}$, and since the exponent of the viscosity law is 0.745 for heptane and 0.5 for toluene, it follows that $G^{*}$ should be proportional to $M^{-\frac{3}{2}}$ in heptane and to $M^{-\frac{4}{3}}$ in toluene. This is what was experimentally observed.

This is again a good argument in favour of a rather regular spatial distribution of the crosslinking sites. Further work is of course necessary to determine the factors which influence the shrinkage or the expansion of the precursor chains upon crosslinking.

It should be added that another argument in favour of the models involving regular distribution of the average position of the branch points in polystyrene networks was obtained recently by Herz and Mathis ${ }^{38}$. They applied $\mathrm{x}$-ray scattering to investigate specially designed gels which carried high electronic density elements in their branch points. Here also evidence for rather well defined intercrosslink distances was found.

\section{CONCLUSION}

In this presentation of some recent results obtained on well-characterized model networks we have put emphasis on their structural and configurational behaviour. There are still many problems to be solved, and yet the assumptions are still rather numerous. We believe, however, that the main point is the rather regular distribution of the crosslinks within the swollen network, leading to some model of spring-suspended beads. Experimental support for this type of structure has already been obtained from $x$-ray scattering data and from the neutron low-angle diffraction peaks we have obtained. 
This type of structure is by no means comparable with the sharp periodicity observed in a crystal structure, since in a gel the fluctuation of a branch point around its equilibrium position is by orders of magnitude greater. But this lattice model helps considerably, especially in the interpretation of equilibrium swelling data and of uniaxial compression measurements; here the 'memory term' characterizes the structure of the gel upon its formation, and it seems obvious that at this stage the functionality of the branch points and their concentration imposes some type of regularity in the location of the crosslinking sites, which remains a fundamental characteristic of a given network. A good argument for this statement is the isotropic dilatation of the network when swollen in various solvents. Further arguments have been obtained from neutron scattering experiments on gels under stress, which will be discussed elsewhere.

It seems thus that at the present stage of the investigations on model networks obtained from a linear, well-defined precursor of sharp molecular weight distribution we may try to interpret the experimental results taking into account more structural regularity than was previously expected.

\section{ACKNOWLEDGEMENTS}

The authors wish to express their deep appreciation to all their co-workers who have taken part in this experimental investigation on model networks, carried out under the direction of Professor H. Benoit-namely, Dr G. Beinert, Mr A. Belkebir-Mrani, Dr J. P. Cotton, Dr D. Decker, Mr R. Duplessix, Miss Chr. Etter, Mr A. Haeringer, Dr J. Higgins, Mr F. Isel, Dr J. Jannink, Dr R. Ober, Mr E. Schuster and Dr P. Weiss.

\section{REFERENCES}

1 J. Seidl, J. Malinsky, K. Dušek and W. Heitz, Advanc. Polymer Sci. 5, 113 (1967).

2 D. J. Walsh, G. Allen and G. Ballard, Polymer, 15, 366 (1974).

3 R. Y. S. Chen, C. U. Yu and J. E. Mark, Macromolecules, 6, 746 (1973).

4 P. Weiss, G. Hild, J. Herz and P. Rempp, Makromol. Chem. 135, 249 (1970).

5 G. Hild and P. Rempp, C.R. Acad. Sci. (Paris), 269, 3379 (1969).

6 J. E. Herz, M. Hert and C. Strazielle, Makromol. Chem. 160, 213 (1972).

7 M. Hert, C. Strazielle and J. Herz, C.R. Acad. Sci. (Paris), 276, 395 (1973).

${ }^{8}$ G. Hild, J. Herz and P. Rempp, Polymer Preprints, 14(1), 601 (1973).

9 G. Beinert, G. Hild and P. Rempp, Makromol. Chem. in press.

10 P. Weiss, J. Herz and P. Rempp, Makromol. Chem. 141, 145 (1971).

11 H. Jacobson and W. H. Stockmayer, J. Chem. Phys. 18, 1600 (1950).

12 J. Herz, A. Belkebir-Mrani and P. Rempp, European Polymer J. 9, 1165 (1973).

13 C. Pinazzi, J. Esnault, G. Lesenyer and A. Pleurdeau, Makromol. Chem. 175, 705 (1974).

14 W. Hopkins, R. H. Peters and R. F. T. Stepto, Polymer, 15, 315 (1974).

15 P. J. Flory, Principles of Polymer Chemistry. Cornell University Press: Ithaca, N.Y. (1953).

16 K. Dušek and W. Prins, Advanc. Polymer Sci. 6, 1 (1969).

17 H. M. James and E. G. Guth, J. Chem. Phys. 15/9, 669 (1947).

18 G. Hild and P. Rempp, C.R. Acad. Sci. (Paris), 271, 1432 (1970).

19 A. Haeringer, G. Hild, P. Rempp and H. Benoit, Makromol. Chem. 169, 249 (1973).

20 G. Hild, A. Haeringer, P. Rempp and H. Benoit, Polymer Preprints, 14/1, 352 (1973).

21 M. Ilavsky and W. Prins, Macromolecules, 3, 415 (1970).

22 G. Ayrey, Chem. Rev. 63, 645 (1963).

23 D. Froelich to be published.

24 C. U. Yu and J. E. Mark, Macromolecules, 6, 751 (1973).

25 J. Barshaw and K. J. Smith, J. Polymer Sci. A2, 1041 (1968).

26 A. Belkebir-Mrani, J. Herz and C. Picot, C.R. Acad. Sci. (Paris), 278, 597 (1974).

27 G. Hild, D. Froelich, P. Rempp and H. Benoit, Makromol. Chem. 151, 59 (1972). 


\section{P. REMPP, J. HERZ, G. HILD AND C. PICOT}

${ }^{28}$ E. J. van de Kraats, Thesis, TH, Delft (1967).

29 R. F. Boyer and R. S. Spenger, J. Polymer Sci. 3, 97 (1948).

30 R. Kuhn and H. J. Cantow, Makromol. Chem. 122, 65 (1969).

31 D. Froelich, D. Crawford, T. Rozek and W. Prins, Macromolecules, 5, 100 (1972).

32 A. J. Chompff, Polymer Preprints, 12, 343 (1971).

33 H. Benoit, D. Decker, J. Higgins, C. Picot, J. P. Cotton, B. Farnoux, G. Jannink and R. Ober, Nature Phys. Sci. 245, 13 (1973).

${ }^{34}$ H. Benoit, J. P. Cotton, D. Decker, B. Farnoux, J. Higgins, G. Jannink, R. Ober, C. Picot and J. des Cloizeaux, Macromolecules, 7. 863 (1974).

35 G. Degenkolbe and H. B. Greiff, Kerntechnik, 15, 437 (1973).

36 H. Benoit, J. P. Cotton, D. Decker, R. Duplessix, G. Jannink, R. Ober, C. Picot and P. Rempp, Microsymposium Prague (1974).

37 H. Benoît, D. Decker, R. Duplessix, C. Picot, P. Rempp, F. P. Cotton, B. Farnoux and G. Jannink, J. Polymer Sci., to be published.

${ }^{38}$ G. Beinert, A. Belkebir-Mrani, J. Herz and A. Mathis, European Polymer J., to be published. 